\title{
Erbanlagen und Lebensstil beeinflussen Rheuma
}

Mit Ausnahme eineiiger Zwillinge nach ihrer Geburt haben wohl keine zwei Menschen auf der Welt das gleiche Immunsystem. Bei entzündlich-rheumatischen Erkrankungen entscheidet die erblich festgelegte Immunausstattung mit darüber, ob eine Rheumaerkrankung ausbricht oder nicht - und wie stark sie ausfällt.

Quelle: Pressemitteilung zum 46. Kongress der Deutschen Gesellschaft für Rheumatologie (DHRh),

19.-22. September 2018, Mannheim
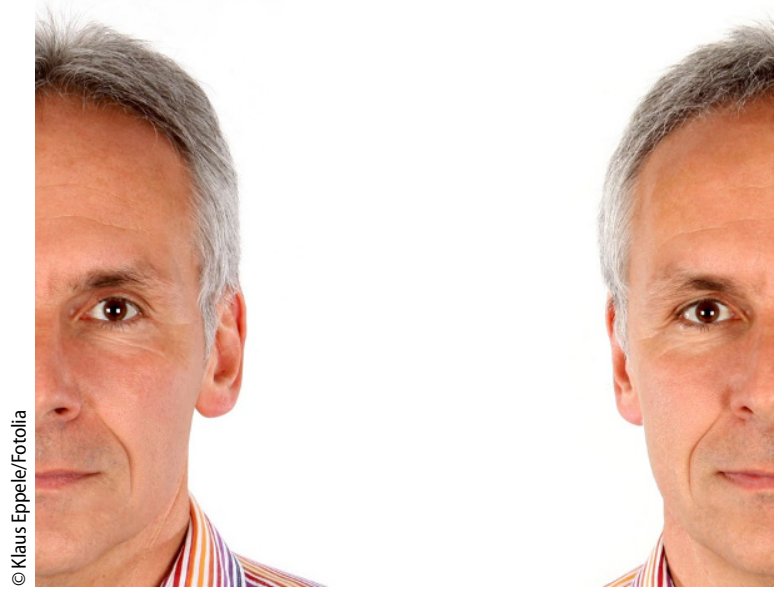

$\Delta$ Zwillingsgeschwister von an Rheuma erkrankten Menschen haben ein deutlich erhöhtes Erkrankungsrisiko; tatsächlich erkranken sie aber in weniger als der Hälfte der Fälle.
Leiter der Sektion Rheumatologie am Universitätsklinikum Heidelberg und Präsident der DGRh.

In den letzten Jahren wurden Zusammenhänge zwischen dem individuellen HLA-Profil und so unterschiedlichen Erkrankungen wie Typ-1-Diabetes, Zöliakie oder entzündlich-rheumatischen Erkrankungen aufgedeckt. Als Beispiel nannte Lorenz Morbus Bechterew, bei dem der rheumatische Entzündungsprozess unter anderem die Gelenke der Wirbelsäule angreift und dazu führt, dass diese versteifen. „95\% der Morbus-Bechterew-Patienten besitzen die Genvariante HLA-B27“, erläuterte Lorenz. In der Gesamtbevölkerung komme diese Variante aber nur zu zehn bis fünfzehn Prozent vor.

\) Raucher haben ein höheres Risiko an Rheuma zu erkranken als Nichtraucher

So deutlich dieser Zusammenhang ist - die Morbus-Bechterew-Forschung belegt auch, dass das Vorhandensein von HLA-B27 alleine nicht ausreicht, um die Krankheit entstehen zu lassen. Denn nur jeder zehnte Genträger erkrankt tatsächlich an Morbus Bechterew. „Offensichtlich sind zusätzliche Faktoren notwendig, damit die Krankheit zum Ausbruch kommt", so Lorenz. Als Auslöser kämen zum Beispiel Infektionen mit bestimmten Viren oder Bak- terien infrage, die das Immunsystem zusätzlich aktivierten und so die Krankheit provozierten.

Auch Studien mit eineiigen Zwillingen belegen die Grenzen des genetischen Einflusses: Zwillingsgeschwister von an Rheuma erkrankten Menschen haben zwar ein deutlich erhöhtes Erkrankungsrisiko. Tatsächlich erkrankt der zweite Zwilling bei den klassischen Autoimmunerkrankungen aber in weniger als der Hälfte der Fälle. Neben Infektionen spielt hier auch der Lebensstil eine Rolle. So haben Raucher zum Beispiel ein höheres Rheumarisiko als Nichtraucher.

"Die Bedeutung des Lebensstils - und damit auch der Eigenverantwortung der Patienten - wird immer deutlicher", sagte Lorenz. Epigenetik ist ein recht neues Forschungsfeld, das die Regulation der Genaktivierung erforscht. Auch hier zeigen sich immer mehr interessante Korrelationen zu entzündlich-rheumatischen Erkrankungen, erläuterte der Experte. Das würde die Hoffnung nähren, dass das Verständnis der Krankheitsgenese auch zu neuen Therapiemöglichkeiten führt.

rheuma plus $2019 \cdot 18: 4$ https://doi.org/10.1007/s12688019-0241-3

(c) Springer-Verlag GmbH Austria, ein Teil von Springer Nature 2019 